\title{
MiR-26a Inhibits Proliferation and Migration of Breast Cancer through Repression of MCL-1
}

\author{
Jie Gao, ${ }^{1,39}$, Laisheng $\mathrm{Li}^{2,39}$, Minqing $\mathrm{Wu}^{1,3}$, Min Liư ${ }^{2}$, Xinhua Xie ${ }^{1,3}$, Jiaoli Guo ${ }^{1,3}$, Hailin Tang ${ }^{1,3}$, \\ Xiaoming Xie ${ }^{1,3 *}$
}

1 Department of Breast Oncology, Sun Yat-sen University Cancer Center, Guangzhou, People's Republic of China, 2 Department of Laboratory Medicine, The First Affiliated Hospital of Sun Yat-sen University, Guangzhou, People's Republic of China, $\mathbf{3}$ State Key Laboratory of Oncology in South China, Sun Yat-sen University Cancer Center, Guangzhou, People's Republic of China

\begin{abstract}
Breast cancer is the most commonly malignancies in women. MicroRNAs are a family of small non-coding RNAs 18-25 nucleotides in length that post-transcriptionally modulate gene expression. MiR-26a has been reported as a tumor suppressor microRNA in breast cancer, which is attributed mainly to targeting of MTDH and EZH2, however, the expression profile and therapeutic potential of miR-26a is still unclear. Here we demonstrate that miR-26a is down-regulated in breast cancer cells and clinical specimens and its modulation in breast cancer cells regulates cell proliferation, colony formation, migration and apoptosis. MCL-1, an anti-apoptotic member of the Bcl-2 family, as novel targets of miR-26a was found to be in reverse correlation with ectopic expression of miR-26a and knockdown of MCL-1 phenocopied the effect of miR-26a in breast cancer cell lines. It was further explored that miR-26a increased sensitivity of breast cancer cells to paclitaxel in which MCL-1 was involved. Thus, miR-26a impacts on cell proliferation and migration of breast cancer by regulating several carcinogenesis-related processes, including a novel mechanism involving the targeting of MCL-1.
\end{abstract}

Citation: Gao J, Li L, Wu M, Liu M, Xie X, et al. (2013) MiR-26a Inhibits Proliferation and Migration of Breast Cancer through Repression of MCL-1. PLoS ONE 8(6): e65138. doi:10.1371/journal.pone.0065138

Editor: Jin Q. Cheng, H. Lee Moffitt Cancer Center \& Research Institute, United States of America

Received December 6, 2012; Accepted April 22, 2013; Published June 4, 2013

Copyright: ( $\odot 2013$ Gao et al. This is an open-access article distributed under the terms of the Creative Commons Attribution License, which permits unrestricted use, distribution, and reproduction in any medium, provided the original author and source are credited.

Funding: This work was supported by funds from Key Program of National Natural Science Foundation of China number 31030061, National Natural Science Foundation of China number 81272514, National Natural Science Foundation of China (31100935), and the China Postdoctoral Science Foundation (2012M520075). The funders had no role in study design, data collection and analysis, decision to publish, or preparation of the manuscript.

Competing Interests: The authors have declared that no competing interests exist.

*E-mail: xiexm@sysucc.org.cn

9 These authors contributed equally to this work.

\section{Introduction}

Breast cancer is the most commonly diagnosed cancer in women and the second leading cause of cancer deaths in the developed world. [1] Although advances in both diagnosis and comprehensive treatment, which incorporates surgery, radiation therapy and chemotherapy, lead to the improvement of prognosis, $[2,3]$ chemotherapy resistance and metastasis remain to be major challenges in breast cancer therapy. [2,3,4,5] Once recurrence or distant relapse occurs due to chemotherapy-resistant or metastatic cells, conventional therapy is nearly ineffective. [5] Therefore, the development of novel effective therapeutic strategies is essential and urgent.

MicroRNAs (miRNA) represent a diverse class of endogenous noncoding RNAs 18-25 nucleotides in length which posttranscriptionally regulate gene expression. [6] Evidence has shown that each miRNA regulates hundreds of target genes [6,7] and $52.5 \%$ of miRNAs are located in cancer-associated genomic regions. [8] Therefore, a large amount of miRNAs are involved in tumorigenesis and function as oncogenes or tumor suppressor genes depending on their targets. [9] Researchers have identified dysregulation of several miRNAs in breast cancer, such as miR-21 [10], miR-34a [11,12], miR-101 [13], miR-122 [14] and miR-155 [15], which contribute to the progression of malignancy. A recent study has suggested miR-26a is downregulated in breast cancer and functions as a tumor suppressor [16], however, the expression profile and therapeutic potential of miR-26a remains unclear.

The global downregulation of miRNAs [17] including miR-26a draws the attention to high-expression genes computationally predicted as putative miR-26a targets in human breast cancer. Apart from MTDH [16] and EZH2 [18], MCL-1(myeloid cell leukemia 1), a pro-survival member of the Bcl-2(B-cell CLL/ lymphoma 2) family, is expected to be highlighted due to the association between the aberrant expression of pro-survival Bcl-2 family proteins and tumorigenesis and resistance to chemotherapeutics [19]. And it has been demonstrated that several miRNAs induces apoptosis by targeting MCL-1 in gastric cancer [20], hepatocellular carcinoma [21], nasopharyngeal carcinoma [22], and multiple myeloma [23].

In this study, we investigated the expression level of miR-26a in breast cancer cells and tissues as well as its effect on cell proliferation, colony formation, migration and apoptosis. Further, we explored the underlying mechanism of miR-26a functions in breast cancer. In addition, we examined the synergistic anti-tumor effects of miR-26a with paclitaxel in breast cancer cells. Our findings will provide a clear understanding of breast cancer pathogenesis as well as suggest that miR-26a may act as a suppressor which can be a novel potential target for breast cancer therapy. 
A

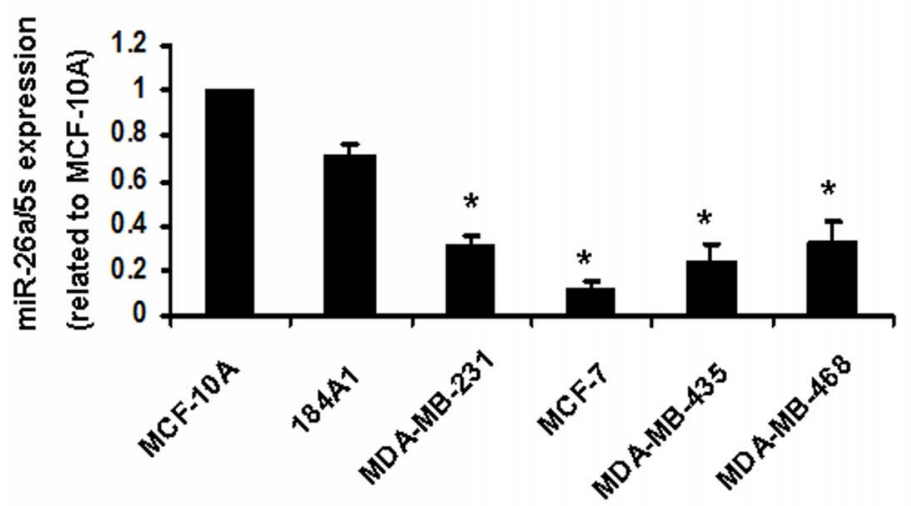

B

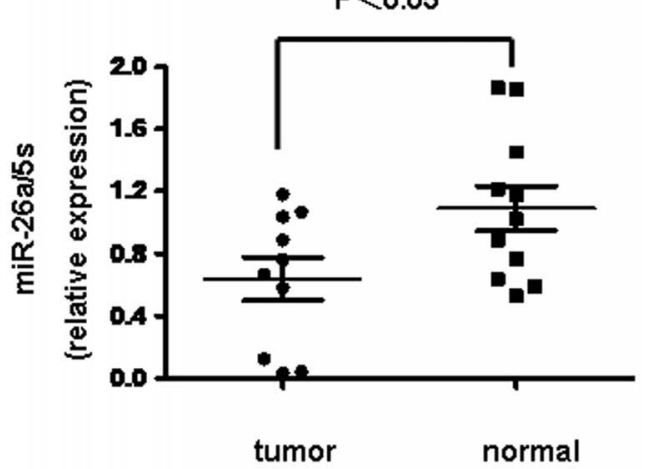

Figure 1. The expression of miR-26a was reduced in breast cancer cell lines and clinical specimens. A. Expression of miR-26a in the 2 immortalized normal mammary epithelium cell lines and 4 breast cancer cell lines. B. Average expression level of miR-26a in human breast cancer tissues and normal breast tissues. MiRNA abundance was normalized to 5s rRNA. ${ }^{*} \mathrm{P}<0.05$ compared with control. doi:10.1371/journal.pone.0065138.g001

Table 1. The relationship between miR-26a expression and clinicopathological parameters in breast cancer.

\begin{tabular}{|c|c|c|c|}
\hline Clinicopathologic parameters & Number of cases & Median expression of $\mathrm{miR}-26 \mathrm{a}$ & $P$-value \\
\hline \multicolumn{4}{|l|}{ Age (years) } \\
\hline$\leq 45$ & 31 & $0.4797 \pm 0.3127$ & 0.135 \\
\hline$>45$ & 21 & $0.5733 \pm 0.2049$ & \\
\hline \multicolumn{4}{|l|}{ Tumor Size $(\mathrm{cm})$} \\
\hline$\leq 2$ & 25 & $0.5608 \pm 0.3276$ & 0.036 \\
\hline$>2$ & 27 & $0.4774 \pm 0.2162$ & \\
\hline \multicolumn{4}{|l|}{ TNM stage } \\
\hline$I+I I$ & 30 & $0.5303 \pm 0.2975$ & 0.409 \\
\hline III+IV & 22 & $0.5000 \pm 0.2492$ & \\
\hline \multicolumn{4}{|l|}{ Lymph node metastasis } \\
\hline No & 27 & $0.5237 \pm 0.3162$ & 0.481 \\
\hline Yes & 25 & $0.5108 \pm 0.2310$ & \\
\hline \multicolumn{4}{|l|}{ ER status } \\
\hline Negative & 22 & $0.5032 \pm 0.2991$ & 0.810 \\
\hline Positive & 30 & $0.5280 \pm 0.2623$ & \\
\hline \multicolumn{4}{|l|}{ PR status } \\
\hline Negative & 28 & $0.5079 \pm 0.2882$ & 0.858 \\
\hline Positive & 24 & $0.5288 \pm 0.2665$ & \\
\hline \multicolumn{4}{|l|}{ Her2 status } \\
\hline Negative & 34 & $0.5491 \pm 0.3134$ & 0.014 \\
\hline Positive & 18 & $0.4578 \pm 0.1785$ & \\
\hline \multicolumn{4}{|l|}{ Ki-67 status } \\
\hline$\leq 20 \%$ & 29 & $0.5607 \pm 0.3197$ & 0.039 \\
\hline$>20 \%$ & 23 & $0.4630 \pm 0.2020$ & \\
\hline \multicolumn{4}{|l|}{ MCL-1 status } \\
\hline Negative & 29 & $0.5734 \pm 0.3224$ & 0.005 \\
\hline Positive & 23 & $0.4470 \pm 0.1865$ & \\
\hline
\end{tabular}

doi:10.1371/journal.pone.0065138.t001 


\section{Materials and Methods}

\section{Clinical Specimens}

Breast cancer biopsy specimens and normal biopsies of the breast were obtained from Sun Yat-sen University Cancer Center (Guangzhou, China), fixed in RNAlater (Ambion, Austin, TX, USA) immediately after biopsy and stored at $-80^{\circ} \mathrm{C}$ until use. Both tumor and normal tissues were histologically confirmed by two different experienced pathologists according to the World Health Organization (WHO) with H\&E (hematoxylin and eosin) staining. Informed consent was obtained from each patient, and the research was approved by the Research Ethics Committee of Sun Yat-Sen University Cancer Center.

\section{Immunohistochemical Staining}

Immunohistochemical staining (IHC) was performed as previously reported [11]. Briefly, paraffin-embedded tissues were sectioned at $5 \mu \mathrm{m}$, deparaffinized, rehydrated through graded alcohols and subjected to antigen retrieval in heated citrate buffer. Following a blocking step, the slides were incubated with primary antibody, washed, biotinylated secondary antibody was applied and the immunocomplexes were visualized using an avidin-biotin complex immunoperoxidase system (Vector Laboratories, Burlingame, CA, USA) with $0.03 \%$ diaminobenzidine (DAB) as a chromagen and hematoxylin as the counterstain. Both external

and internal controls were used to assess the quality of the IHC reaction. For evaluation of the staining the whole area of the section was used. If more than $10 \%$ of the cancer cells contained immunostaining, the patient was classified as MCL-1 positive; if less than $10 \%$ of the cancer cells contained immunostaining, the patient was classified MCL-1 negative. Two pathologists who were blinded to all clinical information scored all specimens. Conflicts (about $5 \%$ of cases) were resolved by consensus.

\section{Cell Culture and miRNA Transfection}

The human breast cancer cell lines MDA-MB-231, MCF-7, MDA-MB-435, MDA-MB-468 and two immortalized normal mammary epithelial cell lines MCF-10A and 184Al were all obtained from American Type Culture Collection, and freshly recovered from liquid nitrogen $(<3$ month). The breast cancer cells were cultured in Dulbecco's modified Eagle's medium (DMED, Invitrogen, CA, USA) supplemented with 10\% fetal bovine serum (FBS, GIBCO, Cappinas, Brazil). MCF-10A cells were cultured in Keratinocyte-SFM (Invitrogen, CA, USA) supplemented with pre-qualified human recombinant epidermal growth factor 1-53 (EGF 1-53, Invitrogen, CA, USA) and bovine pituitary extract (BPE, Invitrogen, CA, USA). 184Al cells were cultured in Mammary Epithelium Basal Medium (MEBM, Clonetics, MD, USA). All cells were cultured in a humidified incubator at $37^{\circ} \mathrm{C}$ containing $5 \% \mathrm{CO}_{2}$.

B

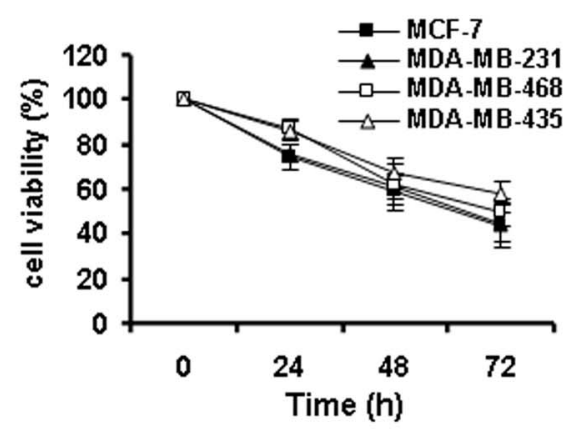

$\operatorname{miR}-26 a(\mu \mathrm{M})$

C.

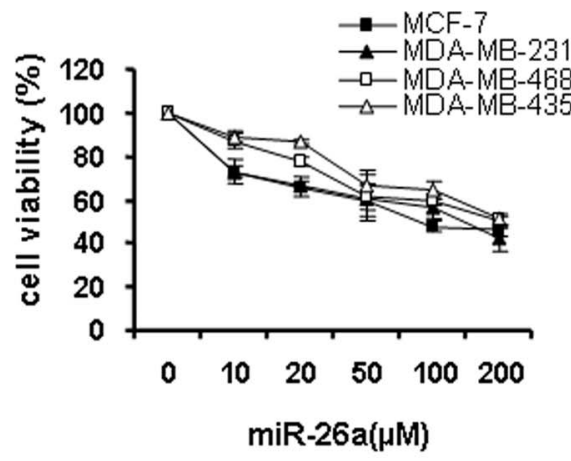

D

miR-Ctrl

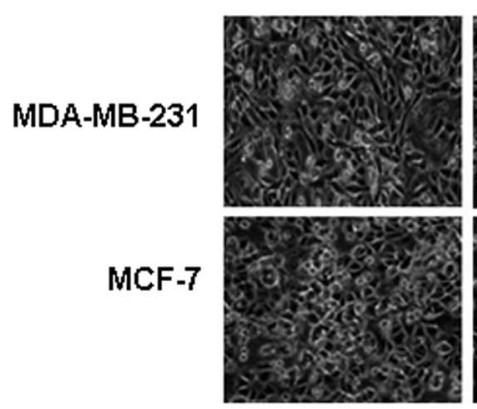

miR-26a

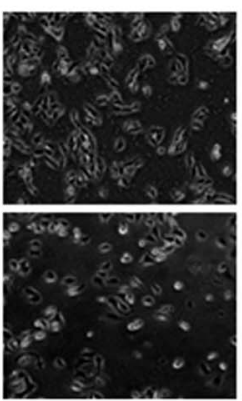

miR-Ctrl miR-26a

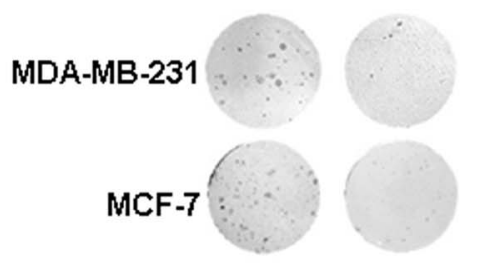

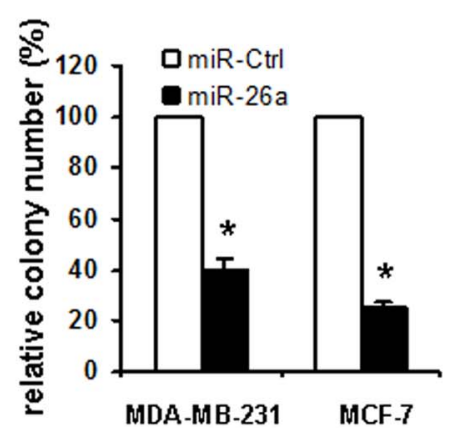

Figure 2. Overexpression of miR-26a lead to reduced cell viability and decreased clonogenicity. A. Dose effect. Cells were transfected with miR-26a at the indicated concentrations for 48 hours. B. Time effect. Cells were transfected with $50 \mu \mathrm{M}$ of miR-26a for indicated periods. C. Morphologic changes of MDA-MB-231 and MCF-7 cells in response to miR-26a inhibition. D. Influence of miR-26a on colony formation of MDA-MB231 and MCF-7 cells. Representative dishes are presented (left). The number of colony was counted for each well of six-well plates and the evaluation of colony numbers was shown in the $y$-axis of the right panel. ${ }^{*} \mathrm{P}<0.05$ compared with control.

doi:10.1371/journal.pone.0065138.g002 
MDA-MB-231

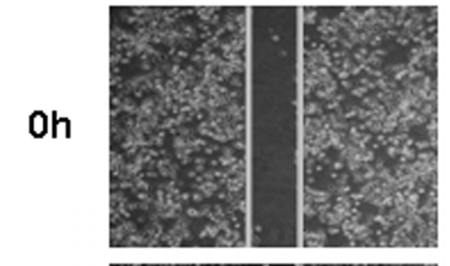

$36 \mathrm{~h}$

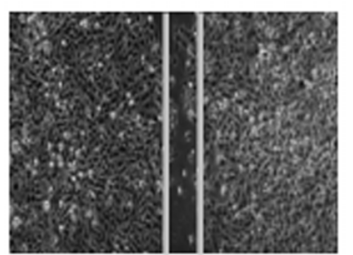

$72 \mathrm{~h}$

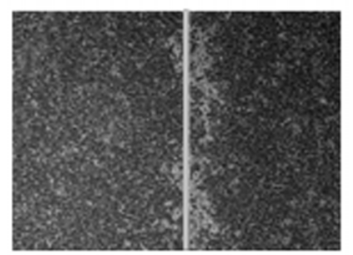

miR-Ctrl
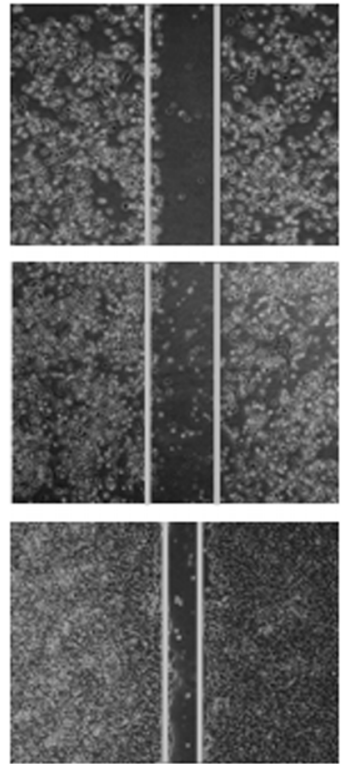

miR-26a

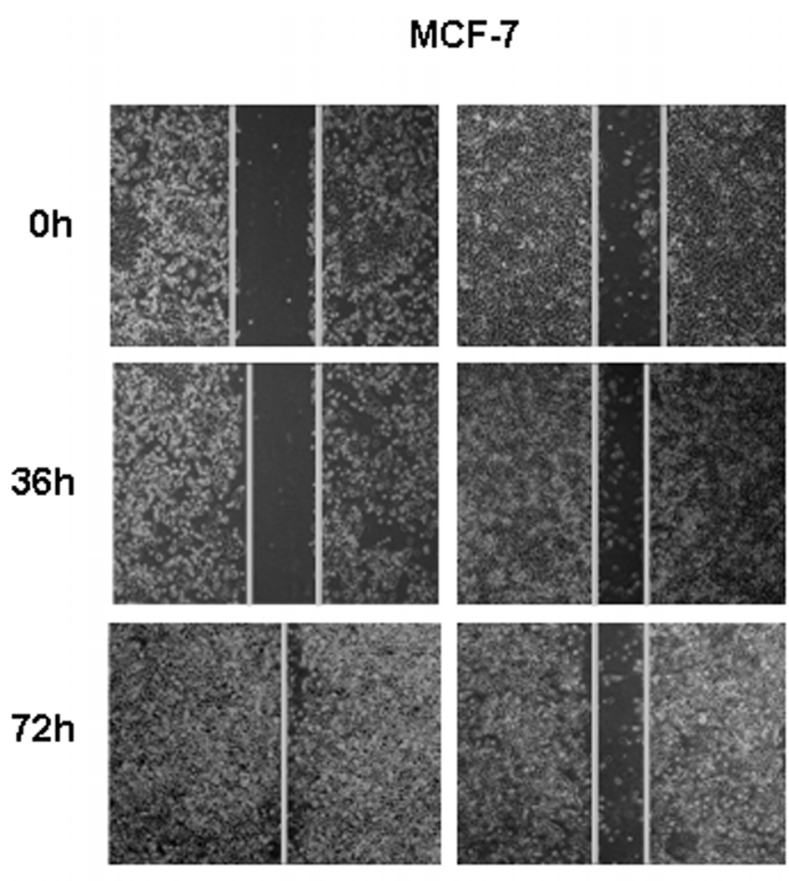

miR-Ctrl
$\operatorname{miR}-26 a$

Figure 3. Ectopic restoration of miR-26a inhibited cell migration. In the wound healing assay, uniform scratches were made in MDA-MB-231 and MCF-7 cells, then serial photographs were obtained at indicated time posttransfection.

doi:10.1371/journal.pone.0065138.g003

The miR-26a mimics and a non-specific miR control (miR-Ctrl) were synthesized and purified by RiboBio (Guangzhou, China). MiRNA mimics were transfected at working concentrations using Lipofectamine 2000 reagent (Invitrogen, CA, USA).

\section{MTT Assay}

Cells were plated in 96 -well plates at $5 \times 10^{3}$ per well in a final volume of $100 \mu \mathrm{L}$ and treated with miRNAs or paclitaxel. After incubation for 24, 48, 72 and 96 hours in a humidified incubator at $37^{\circ} \mathrm{C}$ with $5 \% \mathrm{CO}_{2}, 3-(4,5$-dimethylthiazolyl-2-yl)-2-5 diphenyl tetrazolium bromide (MTT, Sigma, St. Louis, MO, USA) was added. The plates were incubated for another 4 hours, replaced with dimethyl sulfoxide (DMSO, Sigma, St. Louis, MO, USA) after removal of culture medium, and the absorbance was measured at $570 \mathrm{~nm}$ by SpectraMax M5 Microplate Reader (Molecular Deviced, Sunnyvale, CA, USA).

\section{Colony Formation Assay}

Cells were seeded in 6 -well plates at $2 \times 10^{2}$ per well 72 hours after transfection, incubated for 2 weeks. Then the cells were washed twice with PBS, fixed with methanol/acetic acid (3:1, v/v), and stained with $0.5 \%$ crystal violet. The number of colonies was counted under the microscope (Olympus IX81, Tokyo, Japan).

\section{Apoptosis Assay}

Annexin V/propidium iodide (PI) staining was performed for the detection of apoptotic cells. After 48 hours of transfection, $5 \times 105$ cells were collected and washed twice with ice-cold PBS. The cells were then stained using the Alexa Fluor ${ }^{\circledR} 488$ annexin V/ Dead Cell Apoptosis Kit with Alexa ${ }^{\circledR}$ Fluor 488 annexin V and PI for Flow Cytometry (Invitrogen, CA, USA) according to the manufacturer's guidelines. The untreated cells served as a negative control for the double staining. Flow cytometric analysis was performed immediately using a FACSCalibur instrument (Becton Dickinson, CA, USA).

\section{Wound Healing Assay}

MiRNA-transfected cells were scratched using a standard $200 \mu \mathrm{L}$ tip. The debris was removed by washing with serumfree medium. Serial photographs were obtained at different time point using a phase contrast microscope (Olympus IX81, Tokyo, Japan).

\section{RNA Isolation, Reverse Transcription, and Quantitative}

Real-time PCR

Total RNA was extracted using Trizol Reagent (Invitrogen, CA, USA). To quantitate miR-26a expression, reverse transcription was performed with a specific stem-loop real-time PCR miRNA kit (RiboBio, Guangzhou, China). Quantitative real-time PCR (qPCR) was performed using Platinum SYBR Green qPCR SuperMix-UDG system (Invitrogen, CA, USA) on an ABI7900HT System. 5S rRNA was used as an endogenous control. All samples were normalized to internal controls and fold changes were calculated through relative quantification $\left(\mathrm{RQ}=2^{-\Delta \Delta \mathrm{CT}}\right)$.

\section{Western Blot Analysis}

Protein lysates were separated by $10 \%$ SDS-PAGE and electrophoretically transferred to polyvinylidene difluoride membrane (PVDF, Millipore, MA, USA). Then, the membrane was incubated with rabbit polyclonal antibody against human MCL-1 (Santa Cruz, CA, USA) followed by horseradish peroxidase (HRP)-labeled goat-antirabbit IgG (Santa Cruz, CA, USA) and detected by chemiluminescence. Glyceraldehyde-3-phosphate 
miR-Ctrl
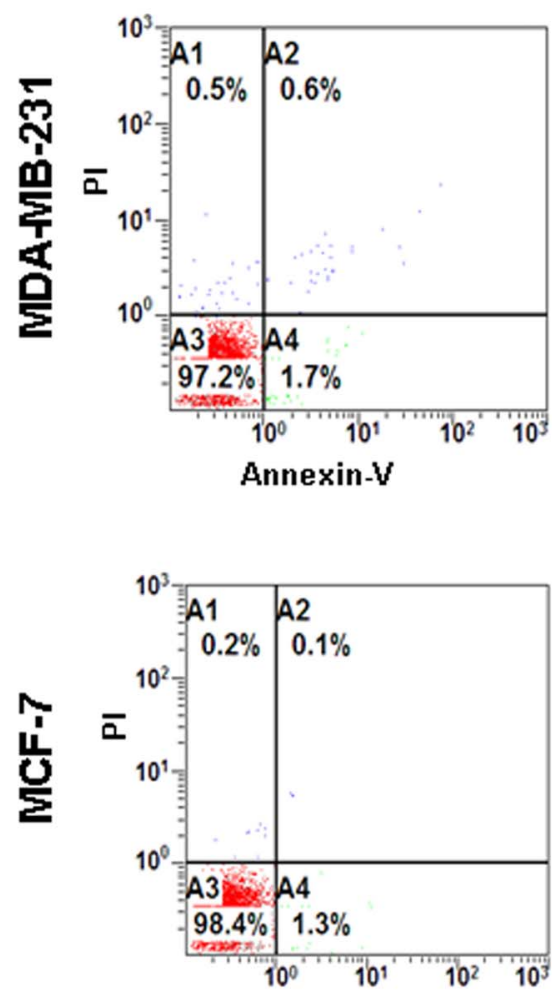

Annexin-V
miR-26a
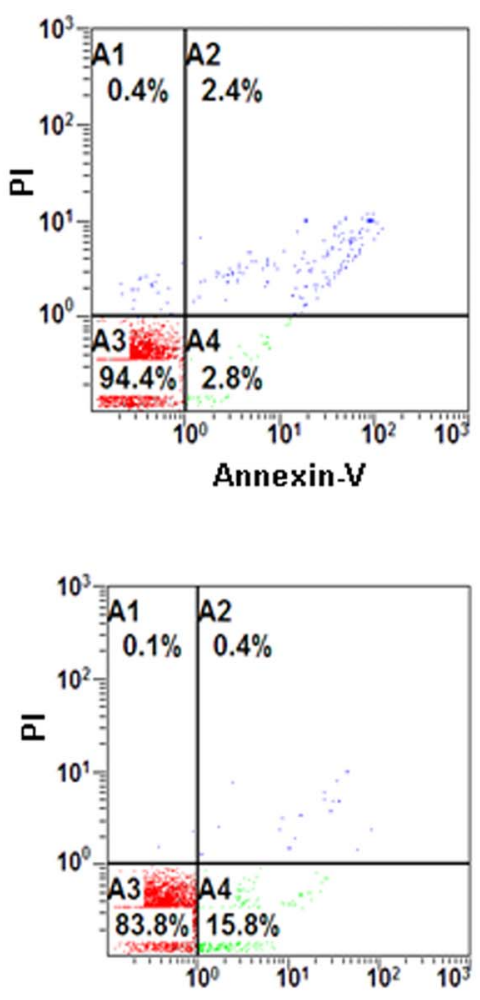
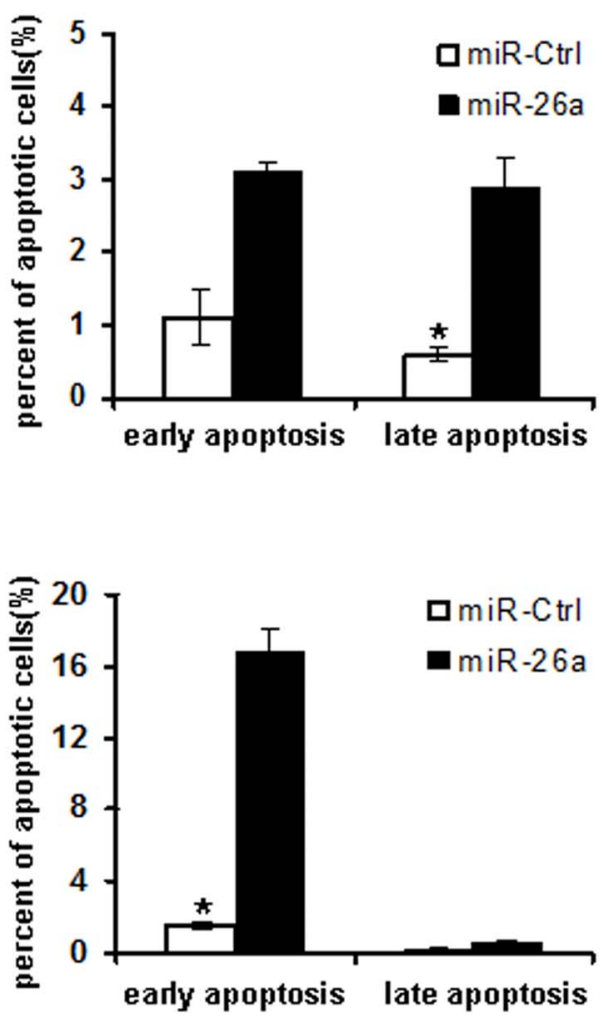

Annexin- $V$

Figure 4. Effect of miR-26a on apoptosis in breast cancer cells. FACS analysis of MDA-MB-231 and MCF-7 cells transfected with miR-26a for 48 hours. The percentage of Annexin V-FITC positive cells to the total cells was shown in the bar graphs. ${ }^{*} \mathrm{P}<0.05$ compared with control. doi:10.1371/journal.pone.0065138.g004

dehydrogenase (GAPDH) was used as a protein-loading control. The specific protein bands were visualized using the Supersignal West Pico ECL chemiluminescence detection kit (Pierce, IL, USA) and Kodak X-ray film (Eastman Kodak Co, NY, USA).

\section{Statistical Analysis}

SPSS 13.0 software was used for stastistical analysis. Data were presented as mean of 3 independent experiments. Two-tailed Student's $t$ test was used for comparisons of 2 independent groups. $P$ values of $<0.05$ were considered statistically significant.

\section{Results}

\section{MiR-26a was Down-regulated in Human Breast Cancer Cell Lines and Clinical Specimens}

The expression of mi-26a was first evaluated in 4 breast cancer cell lines: MDA-MB-231, MCF-7, MDA-MB-435, MDA-MB-468, compared to that of 2 immortal nontumorigenic cell lines MCF$10 \mathrm{~A}$ and 184A1. The result showed that the expression level of miR-26a was decreased significantly in all 4 breast cancer cell lines (Fig. 1A).

We further examined the expression level of miR-26a in 52 breast cancer specimens and 29 normal breast tissues. Similar to the data obtained from the cell lines, the average expression level of miR-26a was significantly lower in breast cancer specimens than in normal breast tissues (Fig. $1 \mathrm{~B} ; \mathrm{P}<0.05)$. In addition, the tumor size of breast cancer patients, HER2 and Ki-67 status are significantly associated with the expression level of miR-26a (Table $1 ; \mathrm{P}<0.05)$.

\section{MiR-26a Reduced Viability and Clonogenicity of Breast \\ Cancer Cells}

To explore the effect of miR-26a on cell growth, MDA-MB231, MCF-7, MDA-MB-435 and MDA-MB-468 cells were transfected with miR-26a mimic. The impact of different doses of miR-26a was evaluated firstly. Cells were transfected with 0 $200 \mu \mathrm{M}$ miR-26a or miR-Ctrl for 48 hours and the relative viability was determined by MTT assay. As shown in Fig. 2A, miR-26a inhibited cell growth in a dose-dependent manner, compared with miR-Ctrl at any of the corresponding concentrations. Next, cells were transfected with $50 \mu \mathrm{M}$ of miR-26a or miRCtrl for varying periods. The result of MTT assay indicated that miR-26a led to growth inhibition in all 4 breast cancer cell lines as early as 48 hours posttransfection, persisting for 72 hours (Fig. 2B).

In addition, the images of MDA-MB-231 and MCF-7 cells demonstrated that miR-26a-transfected cells displayed significantly reduced cell counts, which was concordant with the MTT data. Cells treated with miR-26a exhibited a morphological change with the extension of cytoplasmic portion and rounding of the cell body, while the rest of the spindled cells showed conspicuous shrinkage and extensive detachment (Fig. 2C).

We further performed colony formation assay to determine the effect of miR-26a on clonogenicity of breast cancer cells. As shown in Fig. 2D, miR-26a-transfected MDA-MB-231 and MCF-7 cells 


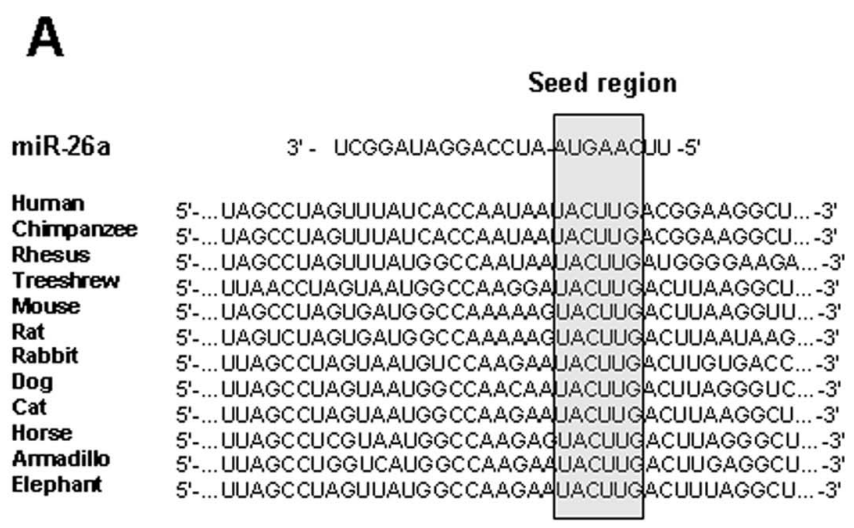

3'UTR of MCL-1 mRNAs

B

MCL-1

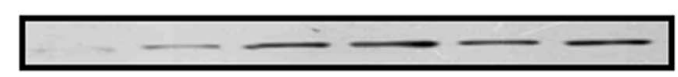

GAPDH

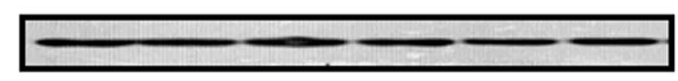

$\begin{array}{lllllll}\text { Relative expression } & 1.00 & 1.87 & 3.22 & 3.83 & 3.39 & 3.87\end{array}$

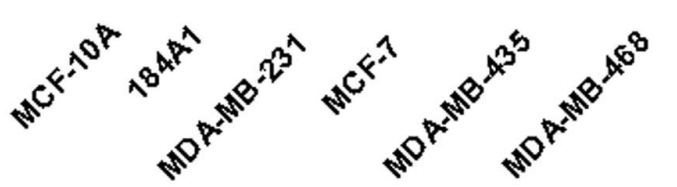

C
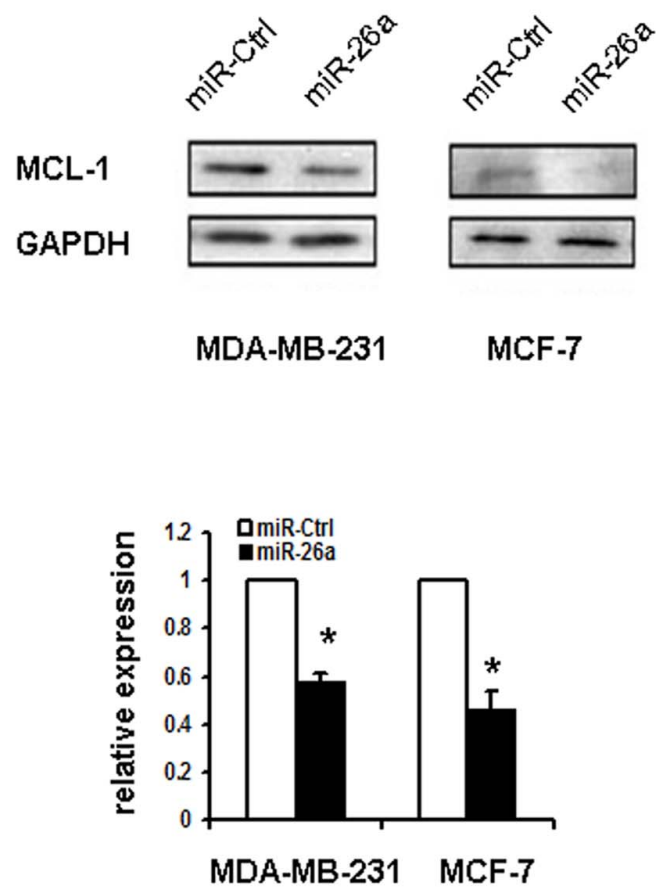

Figure 5. MCL-1 is the target of miR-26a. A. Putative miR-26a binding sites in the $3^{\prime} \mathrm{UTR}$ region of MCL-1 and interspecies conservation of seed matching sequences (gray box). B. Expression of MCL-1 in the 2 immortalized normal mammary epithelium cell lines and 4 breast cancer cell lines. $C$. Western blot assay for MCL-1 expression after MDA-MB-231 and MCF-7 cells were transfected with miR-26a for 48 hours. ${ }^{*} \mathrm{P}<0.05$ compared with control.

doi:10.1371/journal.pone.0065138.g005

exhibited much fewer and smaller colonies compared with miRCtrl-transfected cells $(\mathrm{P}<0.05)$.

\section{MiR-26a Induced Significant Inhibition of Migration in Breast Cancer Cells}

Given that cell migration is among the common functions required by tumor cells for metastatic progression [24], as well as the strong association between tumor cell migration and tumor cell invasion and metastasis [25], we asked whether miR-26a could affect breast cancer cell migration in vitro. In wound healing assay, ectopic restoration of miR-26a did obviously delay the closure rate of both MDA-MB-231 and MCF-7 cells forward the wound area, compared with cells transfected with miR-Ctrl (Fig. 3), which suggested that miR-26a has the ability to inhibit migration for breast cancer cells.

MiR-26a Affected Apoptosis of Breast Cancer Cells in vitro

In order to further investigate the biological effect of miR-26a ectopic restoration on apoptosis in breast cancer cells, we transiently transfected MDA-MB-231 and MCF-7 cells with miR-26a and measured the apoptotic cell death assay using flow cytometric analysis of Annexin V-FITC/PI staining (Fig. 4). The cells in the lower right quadrant of the square chart represent the percentage of early apoptotic cells and in the upper right quadrant represent late apoptotic cells.

Compared to miR-Ctrl, transfection with miR-26a obviously increased the number of early apoptotic cells in MCF-7 (1.5\% vs $16.8 \% ; \mathrm{P}<0.05)$ and late apoptotic cells in MDA-MB-231 $(0.6 \%$ vs $2.9 \% ; \mathrm{P}<0.05)$. There was no significant difference of the number of early apoptotic cells in MDA-MB-231 and late apoptotic cells in MCF-7. These results showed that up-regulation of miR-26a can promote breast cancer cell apoptosis.

\section{MiR-26a Regulated Endogenous MCL-1 Expression in Breast Cancer Cells in vitro}

To figure out the mechanism by which miR-26a may perform in breast cancer, we made an online search of miR-26a targets by Targetscan. Among the hundreds of putative miRNA targets, we focused on MCL-1 as it is an anti-apoptotic member of the Bcl-2 family, which has been validated to be linked with tumorigenesis and resistance to chemotherapy by large amounts of evidences. Target scan showed that there was a miR-26a responsive sequence in $3^{\prime}$-UTR ( $3^{\prime}$ untranslated region) of MCL-1, which was highly conserved among different species (Fig. 5A).

In order to determine whether low expression level of miR-26a is associated with high expression level of MCL-1 in breast cancer cells, we further performed Western blot analyses in the 2 immortalized normal mammary epithelium cell lines and 4 breast 


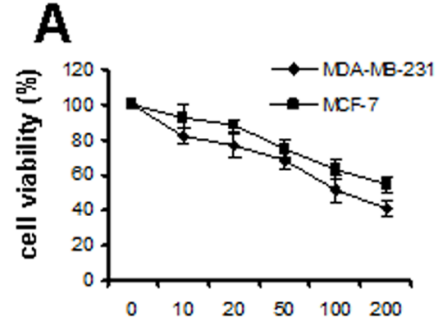

MCL-1-siRNA ( $\mu \mathrm{Mi})$

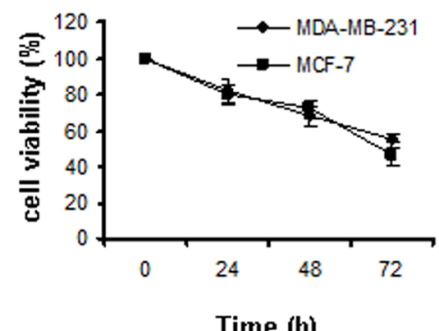

Time (h)

B Ctrl-siRNA MCL-1-siRNA

MDA-MB-231
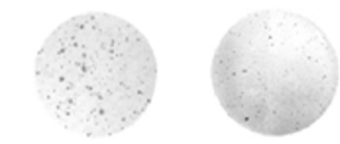

MCF-7
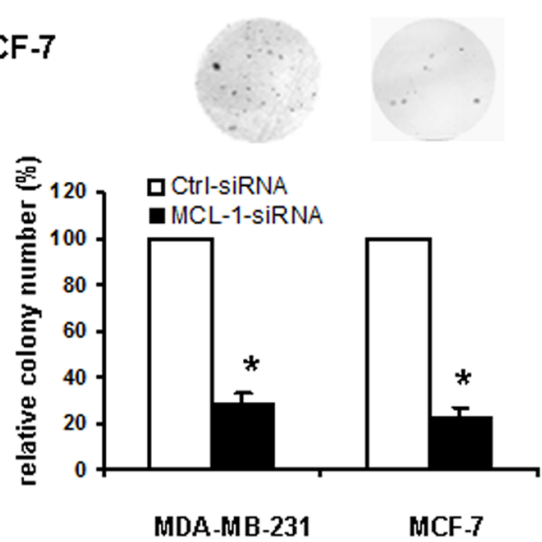
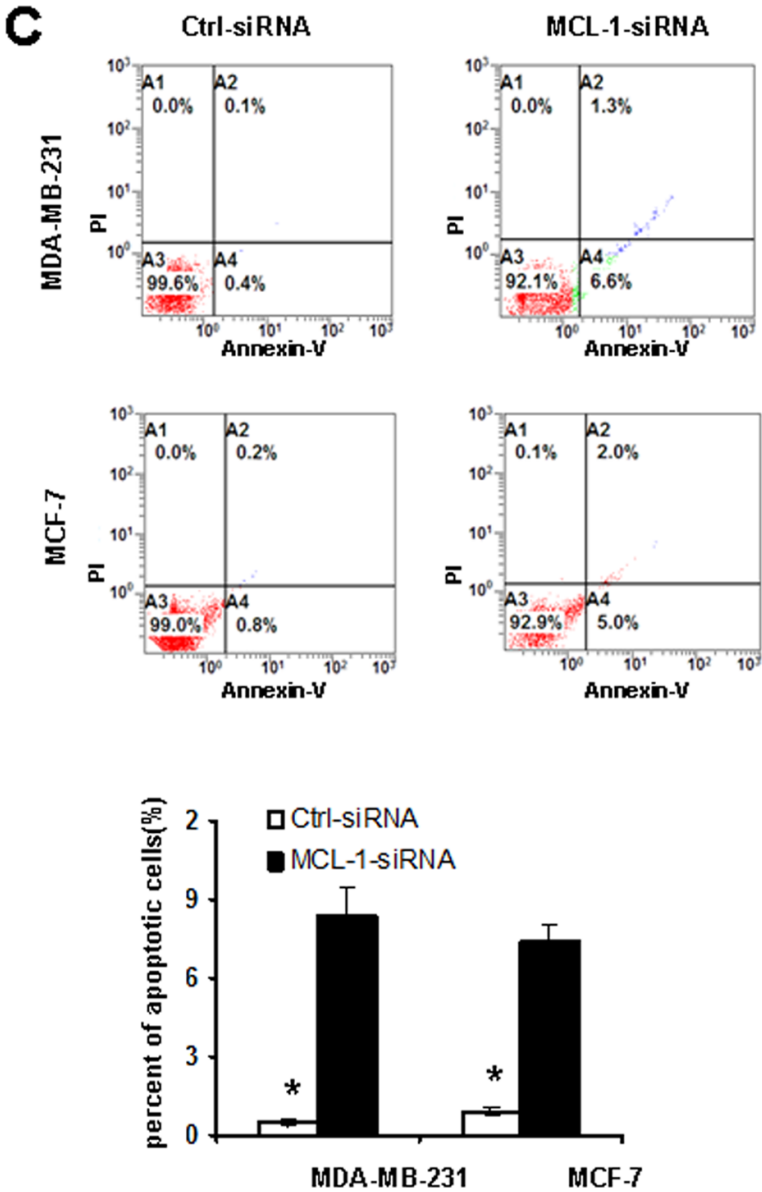

Figure 6. Knockdown of MCL-1 suppresses cell proliferation, clonogenicity and induces cell apoptosis. A. Does effect and time effect of transfection of MCL-1-siRNA on the proliferation of MDA-MB-231 and MCF-7 cells. B. The functional role of MCL-1 in breast cancer cell growth was analyzed by colony formation of MDA-MB-231 and MCF-7 cells. The evaluation of colony numbers was shown in the panel. C. Influence of MCL-1 on apoptosis in breast cancer cells was monitored by flow cytometry. The percentage of Annexin V-FITC positive cells to the total cells was shown in the bar graphs. ${ }^{*} \mathrm{P}<0.05$ compared with control.

doi:10.1371/journal.pone.0065138.g006

cancer cell lines. The result showed that the endogenous MCL-1 expression was obviously increased in all 4 breast cancer cell lines (Fig. 5B). In addition, IHC was carried out to detect MCL-1 expression in breast cancer tissues which were used for miR-26a detected. Compared with the MCL-1 negative group patients, the tumors of the MCL-1 positive group patients had a lower miR-26a expression level (Table $1, \mathrm{P}<0.01)$.

Then we transfected MDA-MB-231 and MCF-7 cells with miR26a or miR-Ctrl and examined MCL-1 expression level by Western blot analyses. As shown in Fig. 5C, the level of MCL-1 protein was significantly down-regulated by transient transfection of miR-26a in both two cell lines $(\mathrm{P}<0.05)$, which means the expression of endogenous MCL-1 was regulated by miR-26a in breast cancer cells.

Knockdown of MCL-1 suppressed cell growth, proliferation and induced cell apoptosis.

In order to address the functional role of MCL-1 in breast cancer cells, MDA-MB-231 and MCF-7 cells were transfected with MCL-1-specific siRNAs. The results of MTT assay indicated that knockdown of MCL-1 led to inhibition of cell growth and proliferation both in these two cell lines (Fig. 6A). A colonyforming assay was carried out to evaluate the effect of MCL-1 on the clonogenicity ability of breast cancer cells. As shown in Fig. 6B, MCL-1-siRNA-transfected cells displayed fewer and smaller colonies compared with control-siRNA transfectants $(\mathrm{P}<0.05)$. Furthermore, flow cytometry analysis revealed that transfection with MCL-1-siRNA significantly increased the number of apoptotic cells in both MDA-MB-231 $(0.5 \%$ vs $8.4 \% ; \mathrm{P}<0.05)$ and MCF-7 (0.9\% vs 7.4\%; P<0.05) cells(Fig. 6C).

\section{MiR-26a Increased Sensitivity to Paclitaxel in Breast Cancer Cells}

Given that paclitaxel was involved in the majority of strategies of conventional chemotherapy, and several miRNAs have been reported to have the synergistic anti-tumor effects with conventional chemotherapy [26], the raised question was whether miR26a could enhance the therapeutic effect of paclitaxel in breast cancer cells. In order to find the answer, MTT assay was performed 72 hours after the treatment of miR-26a and paclitaxel. As shown in Fig. 7A, the combination treatment caused greater inhibition of cell growth, as compared with paclitaxel alone. This result suggested that miR-26a effectively sensitized breast cancer cells to paclitaxel.

To further determine whether MCL-1 was involved in this function of miR-26a, we examined MCL-1 expression level by Western blot analyses in MDA-MB-231 cells. As shown in Fig. 7B, the level of MCL-1 protein was significantly down-regulated by 
A

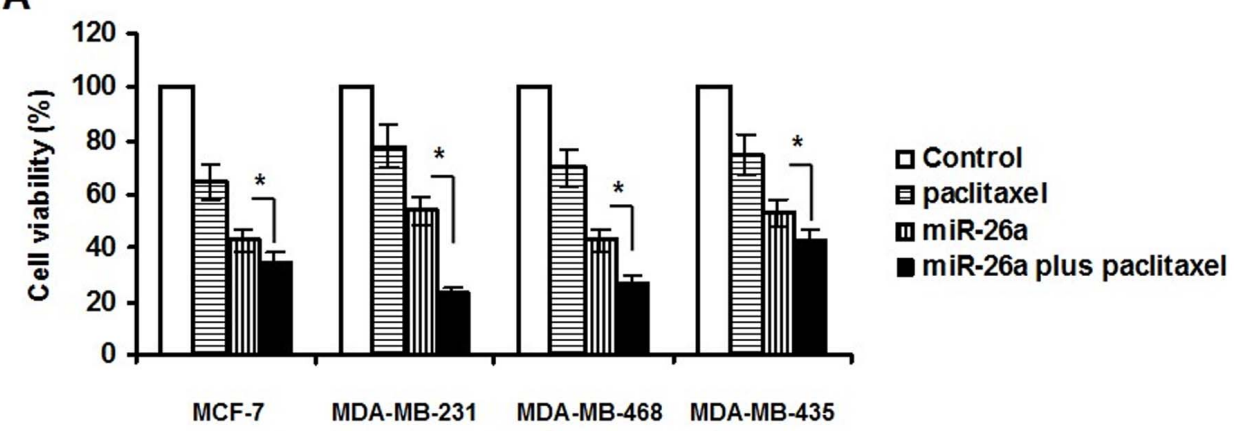

B

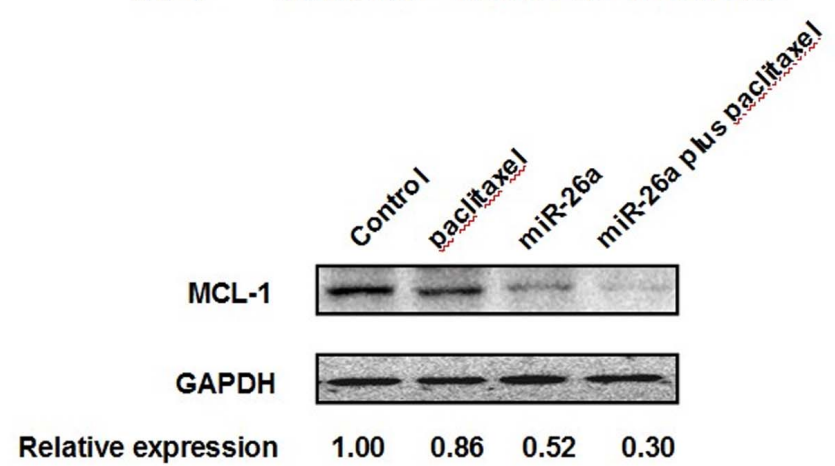

Figure 7. MiR-26a sensitized breast cancer cells to paclitaxel. A. Viability of MDA-MB-231, MCF-7, MDA-MB-435, MDA-MB-468 cells was determined by MTT assay 72 hours after treatment. The percentage of the cell viability as compared with itself without paclitaxel treatment was presented. The concentrations of miR-26a and paclitaxel were $50 \mu \mathrm{M}$ and $0.12 \mathrm{nM}$, respectively. B. Western blot assay for MCL-1 expression 48 hours after treatment in MDA-MB-231 cells. ${ }^{*} \mathrm{P}<0.05$. doi:10.1371/journal.pone.0065138.g007

the combination treatment of miR-26a and paclitaxel, as compared with paclitaxel alone.

\section{Discussion}

Breast cancer is the most frequent cancer of women, which covers $23 \%$ of all cancers [27]. It is a heterogeneous disease due to complicated etiology involving both genetic and environmental factors. Despite the advances of both diagnosis and treatment, breast cancer remains incurable. MiRNAs are a class of multifunctional small molecules that regulate the stability or translational efficiency of targeted messenger RNAs. Mature miRNAs function in the form of RNA-induced silencing complex (RISG) [28]. The miR-RISC leads to base-pairing interactions between microRNAs and the 3'UTR of the target mRNA, which often repress the gene translation or cleave the target mRNA. [29] It is predicted that miRNAs play pivotal roles in regulating wide biological processes, ranging from cell proliferation, apoptosis, cell cycle progression to cancer initiation and metastasis [30,31]. Moreover, the prominent functions of miRNAs in cancer diagnosis, pathogenesis and prognosis have been demonstrated. $[12,32,33]$.

Since the first time was breast cancer reported to be linked with the altered expression of miRNA in 2005 [34], increasing miRNAs were proved to have close relationship with breast cancer. Unlike several most intensively studied miRNAs, such as let-7 and miR$34 \mathrm{a}$, both of which were demonstrated to be tumor suppressor and decreased in majority cancers, the role of miR-26a in distinct cancer cells depends on cellular context. Our research displayed the decreased expression of miR-26a in all of the 4 breast cancer cell lines as well as the clinical specimens of tumor. Meanwhile, the expression level of miR-26a was significantly correlated with tumor size, Her2 and Ki-27 status of breast cancer patients. In this study, to reveal the role of miR-26a in breast cancer cells in vitro, we tested the effects of miR-26a on cell growth. According to the results, miR-26a functioned as the tumor suppressor in breast cancer as it was shown to have the ability to inhibit breast cancer proliferation, colony formation, migration and induce cell apoptosis. In the sense, the role of miR-26a in breast cancer cells is similar to which in hepatocellular carcinoma [35], lung cancer [36], nasopharyngeal carcinoma [37], lymphoma [38], and contrary to which in T-cell lymphoblastic leukemia [39] and glioma [40].

To our knowledge, our study provides the first piece of evidence that miR-26a restoration reduced proliferation and induced apoptosis through repression of MCL-1. In various tumor types, especially in those miR-26a functions as a tumor suppressor, miR26a was demonstrated to inhibit proliferation and colony formation through down-regulation of the histone-lysine $\mathrm{N}$ methyltransferase, EZH2, a global regulator of gene expression. On the other hand, miR-26a was reported to facilitate carcinogenesis through the inhibition of PTEN. Among the predicted miR-26a targets in Targetscan, MCL-1 attracted our attention as it presented highly conservative base-pairing between the $3^{\prime}$ untranslated region of the mRNA in various species and the "seed sequence" located in the 5 ' end of miR-26a, which is essential to determing whether targeting miRNA results in degradation of mRNA or inhibition of translation [41]. More importantly, MCL1 is a pro-survival member of the Bcl-2 family, which has been studied intensively for the past decades owing to their importance in the regulation of apoptosis, tumorigenesis and cellular responses to anti-cancer therapy. Recently investigators determined that MCL-1 was targeted by several miRNAs in various types of cancer. $[42,43]$ Our study revealed that MCL-1 was overexpressed 
in breast cancer cells and ectopic restoration of miR-26a significantly decreased MCL-1 protein expression. In addition, miR-26a expression level was much higher in breast cancer tissues of MCL-1 negative group patients. Furthermore, functional studies by knockdown of MCL-1 phenocopied overexpression of miR-26a in breast cancer cells, resulting in cell growth and proliferation suppression in vitro, suggesting that the growth inhibitory effect of miR-26a was partly implemented by means of repressing MCL-1 expression.

Recently increasing miRNAs have been confirmed possessing potential tumor therapeutic functions [44] as they are involved in tumorigenesis and function as oncogenes or tumor suppressor genes. Therefore, strategies targeting miRNAs expression in cancer would be a new approach to cancer therapy. As we know paclitaxel is used in conventional chemotherapy to treat multiple cancers including breast cancer. Although individuals initially respond favourably, resistance can develop. Surmounting evidences have shown that treatment with paclitaxel alters the expression of genes that confer resistance, while these genes have been confirmed to be regulated by miRNAs [44]. Several research groups reported a novel therapeutic strategy to combine miRNAs gene therapy with chemotherapy. $[45,46]$ While the synergistic anti-tumor effects of several miRNAs with conventional chemotherapy have been reported, $[47,48]$ our research examined the synergistic anti-tumor effects of miR-26a with paclitaxel and the

\section{References}

1. Smith RA, Cokkinides V, Brooks D, Saslow D, Brawley OW (2010) Cancer screening in the United States, 2010: a review of current American Cancer Society guidelines and issues in cancer screening. CA Cancer J Clin 60: 99-119.

2. Chaffer CL, Weinberg RA (2011) A perspective on cancer cell metastasis. Science 331: 1559-1564.

3. Steeg PS (2006) Tumor metastasis: mechanistic insights and clinical challenges. Nat Med 12: 895-904.

4. Rivera E, Gomez H (2010) Chemotherapy resistance in metastatic breast cancer: the evolving role of ixabepilone. Breast Cancer Res 12 Suppl 2: S2.

5. Naumov GN, Townson JL, MacDonald IC, Wilson SM, Bramwell VH, et al. (2003) Ineffectiveness of doxorubicin treatment on solitary dormant mammary carcinoma cells or late-developing metastases. Breast Cancer Res Treat 82: 199206.

6. Bartel DP (2004) MicroRNAs: genomics, biogenesis, mechanism, and function. Cell 116: 281-297.

7. Ambros V (2004) The functions of animal microRNAs. Nature 431: 350-355.

8. Calin GA, Croce CM (2006) MicroRNA signatures in human cancers. Nat Rev Cancer 6: 857-866.

9. Chen CZ (2005) MicroRNAs as oncogenes and tumor suppressors. N EnglJ Med 353: 1768-1771

10. Yan LX, Wu QN, Zhang Y, Li YY, Liao DZ, et al. (2011) Knockdown of miR21 in human breast cancer cell lines inhibits proliferation, in vitro migration and in vivo tumor growth. Breast Cancer Res 13: R2.

11. Li L, Xie X, Luo J, Liu M, Xi S, et al. (2012) Targeted Expression of miR-34a Using the T-VISA System Suppresses Breast Cancer Cell Growth and Invasion. Mol Ther.

12. Li L, Yuan L, Luo J, Gao J, Guo J, et al. (2012) MiR-34a inhibits proliferation and migration of breast cancer through down-regulation of Bcl-2 and SIRT1. Clin Exp Med.

13. Wang R, Wang HB, Hao CJ, Cui Y, Han XC, et al. (2012) MiR-101 Is Involved in Human Breast Carcinogenesis by Targeting Stathmin1. PLoS One 7: e46173.

14. Wang B, Wang H, Yang Z (2012) MiR-122 Inhibits Cell Proliferation and Tumorigenesis of Breast Cancer by Targeting IGF1R. PLoS One 7: e47053.

15. Sun Y, Wang M, Lin G, Sun S, Li X, et al. (2012) Serum MicroRNA-155 as a Potential Biomarker to Track Disease in Breast Cancer. PLoS One 7: e47003.

16. Zhang B, Liu XX, He JR, Zhou CX, Guo M, et al. (2011) Pathologically decreased miR-26a antagonizes apoptosis and facilitates carcinogenesis by targeting MTDH and EZH2 in breast cancer. Carcinogenesis 32: 2-9.

17. Lu J, Getz G, Miska EA, Alvarez-Saavedra E, Lamb J, et al. (2005) MicroRNA expression profiles classify human cancers. Nature 435: 834-838.

18. Wong CF, Tellam RL (2008) MicroRNA-26a targets the histone methyltransferase Enhancer of Zeste homolog 2 during myogenesis. J Biol Chem 283: 9836 9843.

19. Youle RJ, Strasser A (2008) The BCL-2 protein family: opposing activities that mediate cell death. Nat Rev Mol Cell Biol 9: 47-59.

20. Saito Y, Suzuki H, Imaeda H, Matsuzaki J, Hirata K, et al. (2012) The tumor suppressor microRNA-29c is downregulated and restored by celecoxib in human gastric cancer cells. Int J Cancer. result suggested that miR-26a increased sensitivity to paclitaxel in breast cancer cells. Moreover, the study revealed that MCL-1 was involved in this function of miR-26a.

In conclusion, miR-26a was frequently down-regulated in breast cancer tissues and cell lines, which inhibited cell proliferation, colony formation and migration, but promoted apoptosis in breast cancer cells. These effects suggested miR-26a is a potential tumor suppressor in breast cancer. The investigation of the function mechanism indicated that miR-26a inhibited the tumor growth by at least partially targeting MCL-1. Furthermore, miR-26a effectively sensitized breast cancer cells to paclitaxel. The results of our exploration not only suggested the important role of miR26a in breast cancer, but also indicated that therapeutic strategies aimed at restoration of miR-26a expression may be beneficial to patients with breast cancer.

\section{Acknowledgments}

We thank Hongliu Shi for excellent technical assistance.

\section{Author Contributions}

Conceived and designed the experiments: Xiaoming Xie LL. Performed the experiments: J. Gao. Analyzed the data: J. Gao LL MW ML. Contributed reagents/materials/analysis tools: Xinhua Xie J. Guo HT. Wrote the paper: J. Gao LL MW ML.

21. Gong J, Zhang JP, Li B, Zeng C, You K, et al. (2012) MicroRNA-125b promotes apoptosis by regulating the expression of Mcl-1, Bcl-w and IL-6R. Oncogene.

22. Zhang JX, Qian D, Wang FW, Liao DZ, Wei JH, et al. (2012) MicroRNA-29c enhances the sensitivities of human nasopharyngeal carcinoma to cisplatin-based chemotherapy and radiotherapy. Cancer Lett.

23. Zhang YK, Wang H, Leng Y, Li ZL, Yang YF, et al. (2011) Overexpression of microRNA-29b induces apoptosis of multiple myeloma cells through down regulating Mcl-1. Biochem Biophys Res Commun 414: 233-239.

24. Tavazoie SF, Alarcon C, Oskarsson T, Padua D, Wang Q et al. (2008) Endogenous human microRNAs that suppress breast cancer metastasis. Nature 451: $147-152$

25. Entschladen F, Drell TLt, Lang K, Joseph J, Zaenker KS (2004) Tumour-cell migration, invasion, and metastasis: navigation by neurotransmitters. Lancet Oncol 5: 254-258

26. Li Y, VandenBoom TG 2nd, Kong D, Wang Z, Ali S, et al. (2009) Upregulation of miR-200 and let-7 by natural agents leads to the reversal of epithelial-to-mesenchymal transition in gemcitabine-resistant pancreatic cancer cells. Cancer Res 69: 6704-6712.

27. Parkin DM (2001) Global cancer statistics in the year 2000. Lancet Oncol 2: 533-543.

28. Garzon R, Marcucci G, Croce GM (2010) Targeting microRNAs in cancer: rationale, strategies and challenges. Nat Rev Drug Discov 9: 775-789.

29. Cullen BR (2004) Transcription and processing of human microRNA precursors. Mol Cell 16: 861-865.

30. Zhang B, Wang Q Pan X (2007) MicroRNAs and their regulatory roles in animals and plants. J Cell Physiol 210: 279-289.

31. Esquela-Kerscher A, Slack FJ (2006) Oncomirs - microRNAs with a role in cancer. Nat Rev Cancer 6: 259-269.

32. Cummins JM, Velculescu VE (2006) Implications of micro-RNA profiling for cancer diagnosis. Oncogene 25: 6220-6227.

33. Ahmed FE (2007) Role of miRNA in carcinogenesis and biomarker selection: a methodological view. Expert Rev Mol Diagn 7: 569-603.

34. Iorio MV, Ferracin M, Liu CG, Veronese A, Spizzo R, et al. (2005) MicroRNA gene expression deregulation in human breast cancer. Cancer Res 65: 70657070 .

35. Ji J, Shi J, Budhu A, Yu Z, Forgues M, et al. (2009) MicroRNA expression, survival, and response to interferon in liver cancer. N Engl J Med 361: 14371447.

36. Liu B, Wu X, Wang C, Liu Y, Zhou Q et al. (2012) MiR-26a enhances metastasis potential of lung cancer cells via AKT pathway by targeting PTEN. Biochim Biophys Acta 1822: 1692-1704.

37. Lu J, He ML, Wang L, Chen Y, Liu X, et al. (2011) MiR-26a inhibits cell growth and tumorigenesis of nasopharyngeal carcinoma through repression of EZH2. Cancer Res 71: 225-233.

38. Sander S, Bullinger L, Wirth T (2009) Repressing the repressor: a new mode of MYC action in lymphomagenesis. Cell Cycle 8: 556-559. 
39. Mavrakis KJ, Van Der Meulen J, Wolfe AL, Liu X, Mets E, et al. (2011) A cooperative microRNA-tumor suppressor gene network in acute T-cell lymphoblastic leukemia (T-ALL). Nat Genet 43: 673-678.

40. Huse JT, Brennan C, Hambardzumyan D, Wee B, Pena J, et al. (2009) The PTEN-regulating microRNA miR-26a is amplified in high-grade glioma and facilitates gliomagenesis in vivo. Genes Dev 23: 1327-1337.

41. Lewis BP, Burge CB, Bartel DP (2005) Conserved seed pairing, often flanked by adenosines, indicates that thousands of human genes are microRNA targets. Cell 120: 15-20.

42. Mott JL, Kobayashi S, Bronk SF, Gores GJ (2007) mir-29 regulates Mcl-1 protein expression and apoptosis. Oncogene 26: 6133-6140.

43. Crawford M, Batte K, Yu L, Wu X, Nuovo GJ, et al. (2009) MicroRNA 133B targets pro-survival molecules MCL-1 and BCL2L2 in lung cancer. Biochem Biophys Res Commun 388: 483-489.
44. Kasinski AL, Slack FJ (2011) Epigenetics and genetics. MicroRNAs en route to the clinic: progress in validating and targeting microRNAs for cancer therapy. Nat Rev Cancer 11: 849-864.

45. Kojima K, Fujita Y, Nozawa Y, Deguchi T, Ito M (2010) MiR-34a attenuates paclitaxel-resistance of hormone-refractory prostate cancer PC3 cells through direct and indirect mechanisms. Prostate 70: 1501-1512.

46. Holleman A, Chung I, Olsen RR, Kwak B, Mizokami A, et al. (2011) miR-135a contributes to paclitaxel resistance in tumor cells both in vitro and in vivo. Oncogene 30: 4386-4398.

47. Galluzzi L, Morselli E, Vitale I, Kepp O, Senovilla L, et al. (2010) miR-181a and miR-630 regulate cisplatin-induced cancer cell death. Cancer Res 70: 17931803.

48. Kim SJ, Oh JS, Shin JY, Lee KD, Sung KW, et al. (2011) Development of microRNA-145 for therapeutic application in breast cancer. J Control Release 155: 427-434 